\title{
Fluorescent Thiol-Derivatized Gold Clusters Embedded in Polymers
}

\author{
G. Carotenuto, ${ }^{1}$ S. De Nicola, ${ }^{2,3}$ and L. Nicolais ${ }^{4}$ \\ ${ }^{1}$ Institute for Composite and Biomedical Materials, National Research Council, Piazzale E. Fermi 1, 80055 Portici, Italy \\ ${ }^{2}$ Istituto Nazionale di Ottica, CNR, Via Campi Flegrei 34, 80078 Pozzuoli, Italy \\ ${ }^{3}$ INFN Sezione di Napoli, Complesso Universitario di M.S. Angelo, Via Cinthia, 80126 Napoli, Italy \\ ${ }^{4}$ Department of Material Engineering and Production, University of Naples "Federico II", Piazzale Tecchio 80, 80125 Naples, Italy
}

Correspondence should be addressed to S. De Nicola; s.denicola@alice.it

Received 9 April 2013; Accepted 28 May 2013

Academic Editor: Vladimir Tsukruk

Copyright (C) 2013 G. Carotenuto et al. This is an open access article distributed under the Creative Commons Attribution License, which permits unrestricted use, distribution, and reproduction in any medium, provided the original work is properly cited.

Owing to aurophilic interactions, linear and/or planar $\mathrm{Au}(\mathrm{I})$-thiolate molecules spontaneously aggregate, leading to molecular gold clusters passivated by a thiolate monolayer coating. Differently from the thiolate precursors, such cluster compounds show very intensive visible fluorescence characteristics that can be tuned by alloying the gold clusters with silver atoms or by conjugating the electronic structure of the metallic core with unsaturated electronic structures in the organic ligand through the sulphur atom. Here, the photoluminescence features of some examples of these systems are shortly described.

\section{Introduction}

The unique properties of nanosized metal phases $[1,2]$ can be used to fabricate novel classes of technologically useful devices [3-6]. Polymer embedding of metal nanostructures represents a very simple method to handle and use these new types of substances, taking advantage of their physical and catalytic characteristics [7]. These systems are known as nanocomposites, and they have particular importance in the optical field where amorphous polymeric matrices can gain very useful optical characteristics by hosting an adequate metallic phase (e.g., surface plasmon resonance, magnetic properties, radiopacity, etc.) [7].

Atomic metal clusters are ultrasmall metal nanoparticles (diameter lower than two nanometers) and represent the link between atoms and nanocrystals. Metal clusters can show molecular properties like fluorescence. The excellent fluorescence characteristics of molecular gold clusters coated by a monolayer of thiolate molecules are well known [812]. Owing to aurophilic interactions [13], linear $\mathrm{Au}(\mathrm{I})$ thiolate molecules (e.g., $\mathrm{Au}(\mathrm{I})$-alkyl-thiolates, $\mathrm{AuSC}_{n} \mathrm{H}_{2 n+1}$ ) spontaneously aggregate, producing thiol-derivatized gold clusters $\left(\mathrm{Au}_{x}\left(\mathrm{SC}_{n} \mathrm{H}_{2 n+1}\right)\right.$, with $\left.y<x\right)$. This clustering process gives a little amount of disulfide molecules (RS-SR, with $\mathrm{R}=$ $\mathrm{C}_{n} \mathrm{H}_{2 n+1}$ ) as reaction byproduct, according to the following scheme:

$$
x \mathrm{AuSR} \longrightarrow \mathrm{Au}_{x}(\mathrm{SR})_{y}+\frac{(x-y)}{2} \mathrm{RSSR}
$$

Aurophilic interactions can be considered as a sort of super Van der Waals interactions since an electron correlation of the closed-shell components is involved in their interaction, with a bond energy of ca. $50 \mathrm{kj} / \mathrm{mol}$ [13]. Usually, a mild thermal treatment at temperatures ranging from $80^{\circ} \mathrm{C}$ to $140^{\circ} \mathrm{C}$ is required to convert the oligomeric $\mathrm{Au}(\mathrm{I})$ thiolate molecules to thiol-derivatized gold clusters [8]. The electronic transition involved in the fluorescence of thiolderivatized gold clusters is attributed to ligand-to-metalmetal charge transfer (LMMCT) from sulfur atom in the thiolate ligands to the Au atoms [14]. Gold thiolate crystals have a polymeric structure with sulphur atoms bridging the gold atoms and can generate a fluorescence depending on the concentration of S-Au-Au fluorophores groups present in the system. However, gold thiolate crystals contain only a very limited number of S-Au-Au groups, and, consequently, 


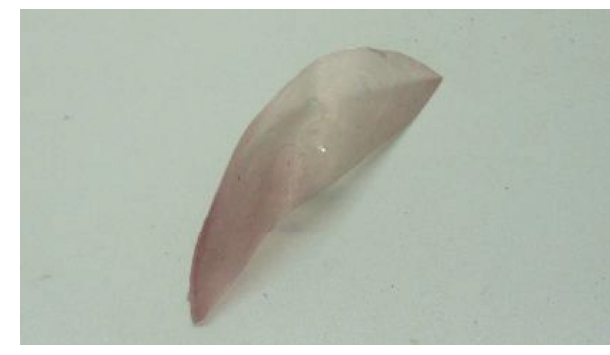

(a)

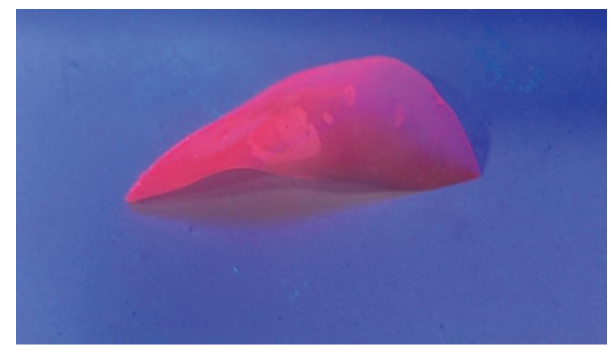

(b)

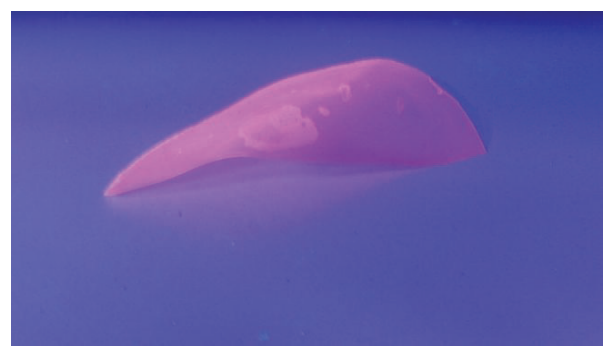

(c)

FIGURE 1: Au (dodecanoic acid)-thiolate clusters embedded into a polyvinyl alcohol (PVA) matrix (1\% by weight) observed under visible light (a), $365 \mathrm{~nm}$ ultraviolet light (b), and $254 \mathrm{~nm}$ ultraviolet light (c).

the fluorescence emission is quite weak. These polymeric $\mathrm{Au}(\mathrm{I})$-thiolate structures can be treated by strong ligand molecules (e.g., N-methyl-imidazole), according to the following ligand-exchange reaction:

$$
\begin{aligned}
& \left(\mathrm{Au}-\mathrm{S}-\mathrm{C}_{12} \mathrm{H}_{25}\right)_{n}+n \mathrm{C}_{4} \mathrm{H}_{6} \mathrm{~N}_{2} \\
& \quad \longrightarrow n \mathrm{Au}\left(\mathrm{C}_{4} \mathrm{H}_{6} \mathrm{~N}_{2}\right)\left(\mathrm{SC}_{12} \mathrm{H}_{25}\right)
\end{aligned}
$$

which leads to a full decomposition of the polymeric thiolate into single monomeric unities and to an increase of the $\mathrm{Au}(\mathrm{I})$ thiolate solubility in organic solvents with a consequent quenching of the weak fluorescence signal. The fluorescence characteristics of thiol-derivatized gold clusters differ significantly from those of ordinary organic fluorophores. The high concentration of S-Au-Au fluorophore groups in the cluster system gives rise to strong fluorescence emission (quantum yield up to ca. 20\%) [8]. In ordinary organic fluorophores (e.g., fluorescein, quinine, rhodamine, etc.), which are also dye chromophores, the color changes upon exposure to ultraviolet or to visible light and the corresponding Stokes shift is relatively short, of the order of few tens of nanometers. On the contrary, thiol-derivatized gold clusters have large Stokes shift of several hundred nanometers. The cluster absorption is completely located in the ultraviolet spectral range (near or far UV), and the fluorescence emission is at greater wavelength in the visible spectrum. Consequently, thiol-derivatized gold clusters are colorless and perfectly transparent under visible light because their small size does not allow for light scattering phenomenon. Due to these peculiar fluorescence properties, thiol-derivatized gold clusters (Au clusters for short) can be employed in applications that can benefit from the use of particles that emit detectable fluorescence. For example, they can be used to fabricate "invisible inks". Indeed, there are a variety of substrates (polymers, textiles, paper, wood, etc.) that can be invisibly treated by polymer-embedded Au clusters and the fluorescence signal can be revealed only by illuminating them by UV-light. Furthermore, only small amounts of fluorophores are required to make these plastic matrices able to generate a detectable visible-light emission (see Figure 1).

Usually, the absorption spectrum is characterized by multiple absorption maxima, and a change of the excitation wavelength results in a modification of the emission color (LEDs are very simple monochromatic UV sources; consequently, the color of emitted radiation can be simply modified by changing the light source type). The possibility of tuning the emission frequency by changing the size of the emitting source is a well-known effect in semiconductor quantum dots, but it cannot be exploited to tune the fluorescence emission of thiol-derivatized Au clusters, because it would require changing in controllable manner the size of the cluster at molecular level. To change the emission frequency of thiol-derivatized Au clusters, one needs to pursue other approaches, such as alloying Au clusters with silver atoms, which have comparable ionic size and crystallize with close crystallographic parameters. Another possibility is coating $\mathrm{Au}$ clusters with aromatic molecules (e.g., imidazole) that conjugate with the Au metal core through the sulfur atom, thus modifying the electronic structure and the fluorescence properties of the whole system.

In this paper we report our investigations on thiolderivatized gold clusters $\left(\mathrm{Au}_{x}\left(\mathrm{SC}_{12} \mathrm{H}_{25}\right)_{y}, \mathrm{Au}_{x}\left(\mathrm{SC}_{14} \mathrm{H}_{29}\right)_{y}\right.$, and $\mathrm{AuSC}_{4} \mathrm{H}_{5} \mathrm{~N}_{2}$, AuMMI). We have developed a simple synthesis protocol for producing these Au-thiolate complexes by dissolving the gold thiolate into amorphous polystyrene, using a solvent-based method, and then heating the obtained 


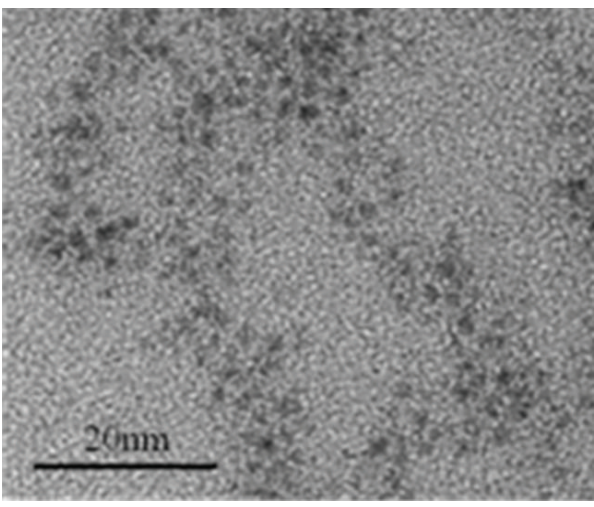

(a)

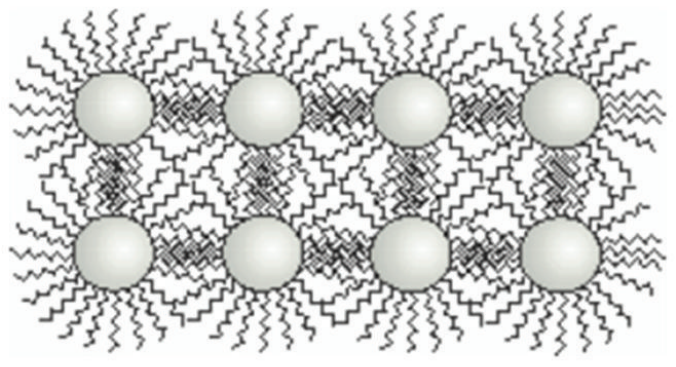

(b)

FIGURE 2: TEM-micrograph of dodecyl-thiolate derivatized Au clusters (a) and schematic representation of the aggregate formation by the alkyl-chain interdigitation process (b).

blend at mild temperatures. The most significant finding is the strong fluorescence emission of the polymer-embedded thiol-derivatized clusters. These materials have been characterized by transmission electron microscopy (TEM), X-ray diffraction (XRD), and optical emission spectroscopy.

\section{Experimental Section}

Thiol-derivatized gold clusters were generated by thermally annealing linear or planar $\mathrm{Au}(\mathrm{I})$-thiolate molecules in the form of both pure-phase or polymeric solutions (amorphous polystyrene), using a thermoblock apparatus [15, 16]. We report on the preparation of gold dodecyl-thiolate (i.e., $\mathrm{AuSC}_{12} \mathrm{H}_{25}$ ), gold tetradecyl-thiolate (i.e., $\mathrm{AuSC}_{14} \mathrm{H}_{29}$ ), and gold 2-mercapto-1-methyl imidazole $\left(\mathrm{AuSC}_{4} \mathrm{H}_{5} \mathrm{~N}_{2}\right)$. The silver terdodecyl-mercaptide (AgTDM) used as alloying metal was provided by Cabro S.p.A. and used without purification.

Gold dodecyl-thiolate was synthesized by treating an ethanol solution of gold tetrachloroauric acid $\left(\mathrm{HAuCl}_{4}\right.$, Aldrich) with an ethanol solution of 1-dodecanethiol $\left(\mathrm{C}_{12} \mathrm{H}_{25} \mathrm{SH}\right.$, Aldrich) at room temperature. The obtained light-yellow precipitate was separated by filtration and dissolved/dispersed in a chloroform solution of amorphous polystyrene (Aldrich, $M_{w}=230,000 \mathrm{gmol}^{-1}$ ). The polymeric films were prepared by casting this solution into a Petri dish and then allowing it to dry in air at room temperature. Usually, self-standing films with thickness of ca. $100 \mu \mathrm{m}$ were obtained. The synthesis of gold tetradecyl-thiolate was carried out by the same chemical route. $\mathrm{Au}(\mathrm{I})$-thiolate was quite soluble in chloroform because it did not organize in layered polymeric structures like those observed in many metal thiolate compounds. This was also supported by the absence of low-angle periodicity in the thiolate XRD. A little amount of $\mathrm{Au}(\mathrm{I})$-thiolate was placed in a glass tube and heated at $140^{\circ} \mathrm{C}$ by a thermoblock in order to develop good fluorescence properties.

Nontoxic, odorless, and highly soluble 2-mercapto-1methylimidazole $\left(\mathrm{C}_{4} \mathrm{H}_{6} \mathrm{~N}_{2} \mathrm{~S}\right.$, Aldrich) reacted in stoichiometric amount with tetrachloroauric acid $\left(\mathrm{HAuCl}_{4}\right.$, Aldrich) in acetonitrile at room temperature under magnetic stirring for 2 days according to the following reaction mechanism:

$$
\begin{aligned}
3 \mathrm{C}_{4} \mathrm{H}_{5} \mathrm{~N}_{2}-\mathrm{SH}+\mathrm{HAuCl}_{4} & \\
& \longrightarrow \mathrm{C}_{4} \mathrm{H}_{5} \mathrm{~N}_{2}-\mathrm{SAu}+\left(\mathrm{C}_{4} \mathrm{H}_{5} \mathrm{~N}_{2} \mathrm{~S}\right)_{2}+4 \mathrm{HCl}
\end{aligned}
$$

The solution appeared bloody-red colored due to the gold-imidazole complex formation and no precipitate was observed. However, after quick addition of a few drops of $1 \mathrm{M} \mathrm{NH}_{4} \mathrm{OH}$ solution until $\mathrm{pH} 7$, under vigorous stirring, the precipitation of the white thiolate salt occurred (the ammonia removed the acid sulfhydryl proton, causing the $\mathrm{Au}(\mathrm{I})$-thiolate formation). The as precipitated material was subjected to a qualitative fluorescence analysis by irradiation with an UV-lamp operating at $\lambda$ of $365 \mathrm{~nm}$. At the beginning, only a low intensity light-blue fluorescence was detectable. After a few hours of digestion at room temperature under stirring, the fluorescence color tuned and a significant green emission was observed. Therefore, the as precipitated thiolate slowly converts to thiol-derivatized gold clusters at room temperature under stirring. The white solid precipitate was first isolated by vacuum filtration (Whatman GF/C Filters), accurately washed with $20 \mathrm{~mL}$ of acetonitrile, and then dried in air at room temperature. The alloyed $\mathrm{Au} / \mathrm{Ag}$ clusters were produced by mild heating of an heptane solution of AuSC $_{14} \mathrm{H}_{29}$ and AgTDM (silver terdodecyl-mercaptide) for 1 hour at $80^{\circ} \mathrm{C}\left(\right.$ AuSC $_{14} \mathrm{H}_{29}: \operatorname{AgTDM}=1: 9$ by weight $)$ in a thermostatic bath under stirring, following the same isolation/purification procedure as for the AuMMI product.

\section{Results and Discussion}

The microstructure of dodecyl-thiolate derivatized Au clusters was investigated by transmission electron microscopy (TEM). As visible in the TEM micrograph shown in Figure 2(a), these nanostructures have a very small size (the measured diameter was of ca. 1.8-2.4 nm), quite monodispersed, with a pseudospherical shape, and have a strong 


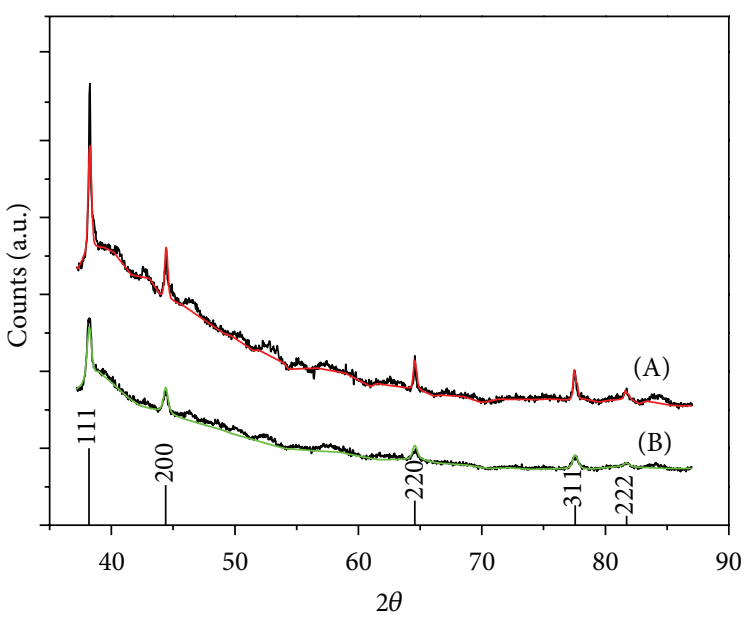

(a)

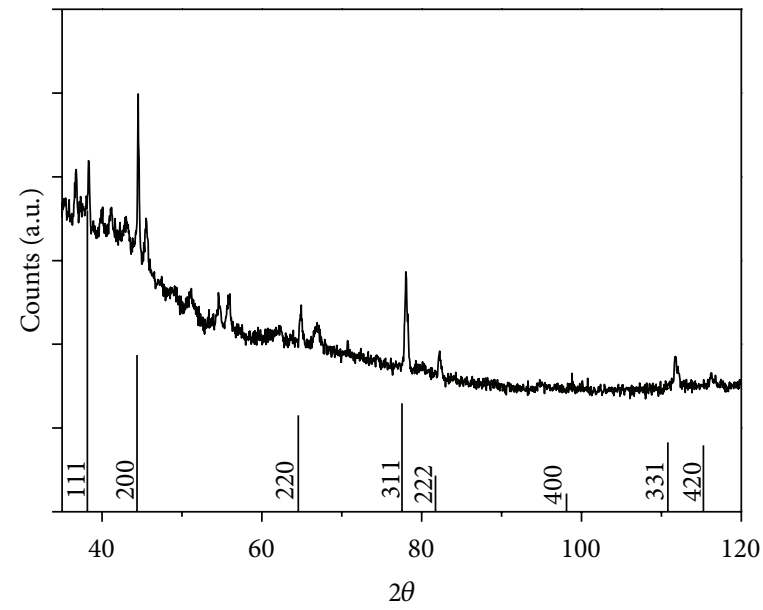

(b)

FIGURE 3: XRD of gold clusters embedded in amorphous polystyrene before (A) and after (B) purification by film dipping in pure acetone (left-side picture) and XRD ( $\theta-2 \theta$ run) of the AuMMI/PS system (right-side picture). The red and green lines in Figure 3(a) are the Rietveld best-fitted profiles of the experimental data.

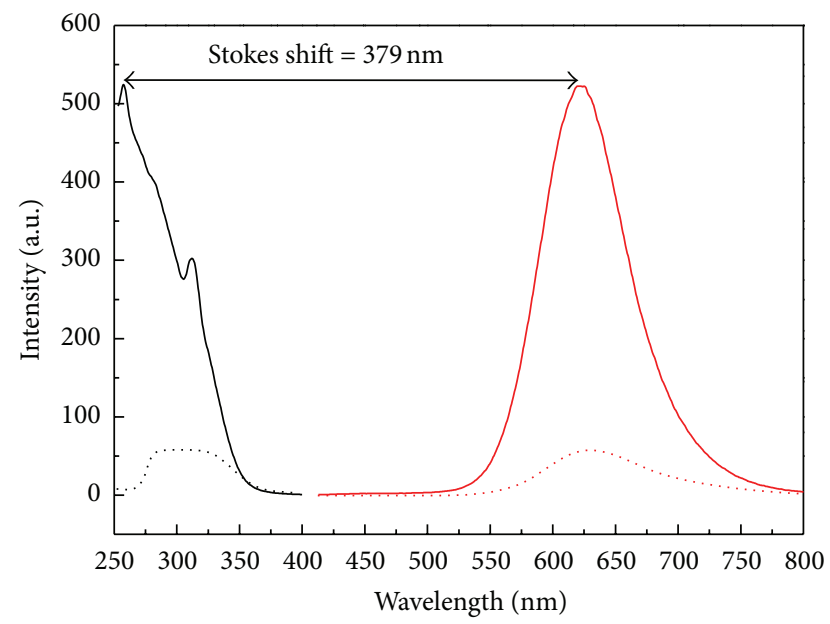

(a)

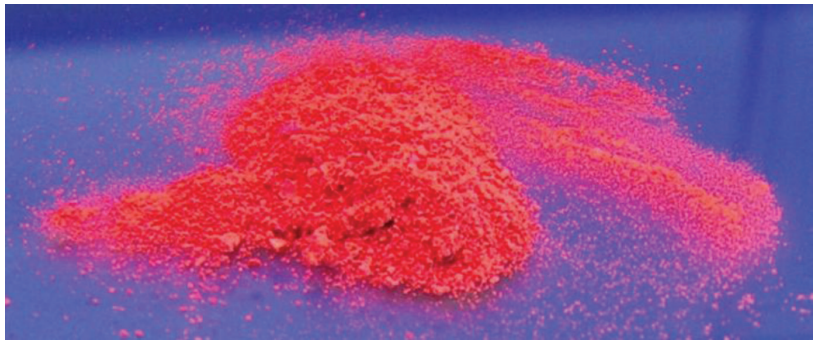

(b)

FIGURE 4: Photoemission spectra (red lines) of the luminescent polymer-embedded Au clusters obtained from linear Au-thiolates: dodecylthiolate (dotted line); tetradecyl-thiolate (solid line) (a). Pure gold dodecyl-thiolate powder under UV-light (365 nm) (b).

tendency to form aggregates. Such variously shaped and sized aggregates are probably generated in the amorphous polystyrene matrix because of the possibility for the linear alkyl chains present at cluster surface to interdigitate and crystallize together (see schematic representation of the aggregate in Figure 2(b)).

The XRD of Au clusters derivatized by dodecyl-thiolate embedded into an amorphous polystyrene matrix is shown in Figure 3(a). This sample shows a crystalline nature with peaks located at $2 \theta$ values of $38.2^{\circ}, 44.4^{\circ}, 64.6^{\circ}, 77.5^{\circ}$, and $81.7^{\circ}$ that correspond to the diffraction pattern of pure metallic gold in the face-centered cubic (FCC) structure. The extremely small size of metal crystallites produces broad diffraction peaks of very low intensities. The constant lattice value estimated from the XRD is found to be $4.08 \AA$, and the average size calculated by the Scherrer equation was of $2.8 \pm 0.3 \mathrm{~nm}$. The XRD baseline is not flat because the gold diffraction pattern overlaps the polystyrene diffuse alone; in addition this material contains additional crystalline phases (probably disulfide, R-S-R, crystals, and the interdigitated crystalline regions) that increase the baseline noise.

In order to remove all the organic crystalline phases from the system, the films were dipped for $24 \mathrm{~h}$ in acetone and then dried in air at room temperature. The purification process resulted in a substantial improvement of the XRD of the purified sample, as can be seen by comparing the XRD in Figure 3(a). Both diffractograms contain also the Rietveld best-calculated profile (red and green lines) for comparison 


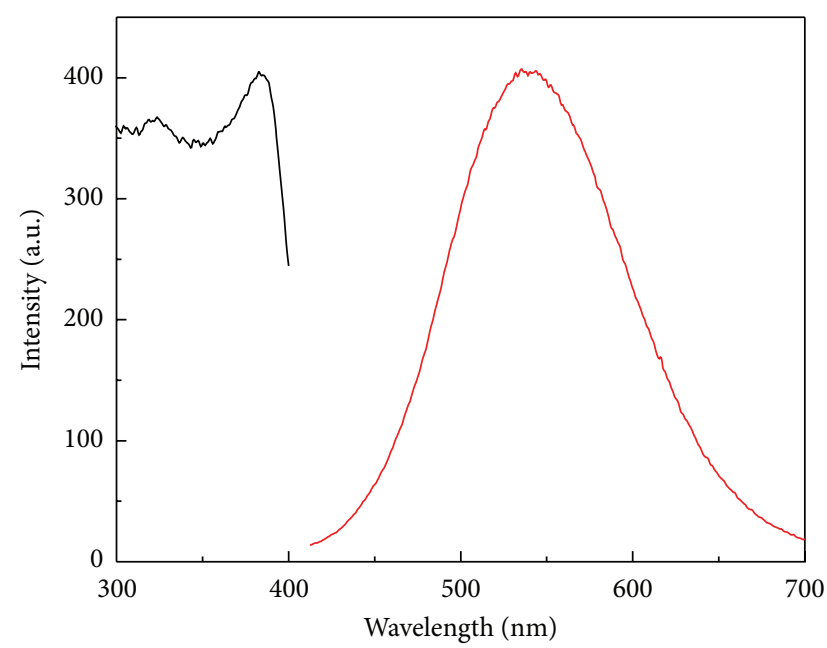

(a)

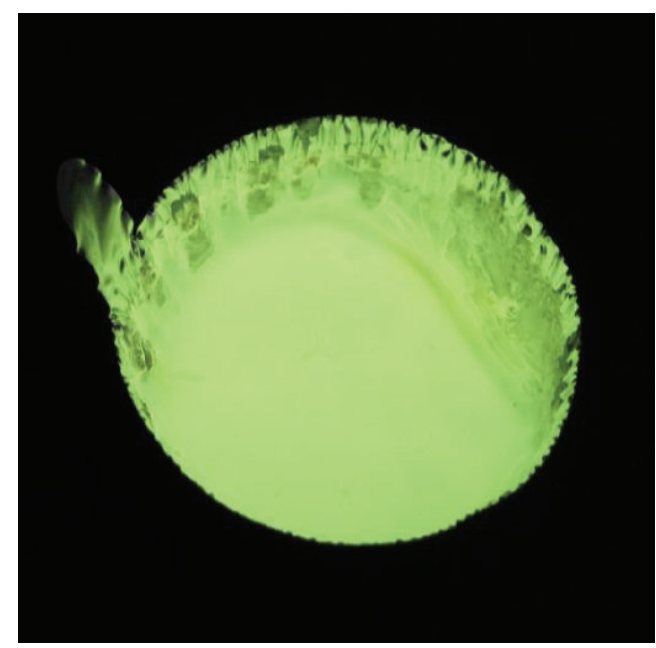

(b)

FIgure 5: Polystyrene-embedded AuMMI clusters: PL spectrum (a), and sample (3\% by weight of AuMMI) under UV-light (365 nm) (b).

with the experimental data (goodness of fit is 3.1). Figure 3(b) shows the diffraction pattern of the pure AuMMI powder dispersed in amorphous polystyrene $(\theta-2 \theta$ run).

The fabricated plastic films were colourless under visible light and quite transparent, but they were found to emit strong fluorescence upon exposure to short-wave $(254 \mathrm{~nm})$ and long-wave $(365 \mathrm{~nm})$ UV-light. The emission colour of the fluorescence was determined by the type of embedded clusters and was found to range from yellow-green to orangered.

Figure 4 shows the photoluminescent (PL) spectra of the polymer-embedded Au clusters obtained by mild thermal treatment of $\mathrm{n}$-alkyl thiolates (i.e., dotted-lines for AuSC $_{12} \mathrm{H}_{25}$ and solid lines for AuSC ${ }_{14} \mathrm{H}_{29}$ precursors). The $\mathrm{Au}_{x}\left(\mathrm{SC}_{14} \mathrm{H}_{29}\right)_{y}$ clusters spectrum exhibits a strong emission peak centred at wavelength $\lambda=633 \mathrm{~nm}$. This emission spec-trum was obtained for an excitation wavelength $\lambda_{\text {exc }}=$ $254 \mathrm{~nm}$ which corresponds to an excitation maximum, and the corresponding Stokes shift is $\Delta \lambda=379 \mathrm{~nm}$. The $\mathrm{Au}_{x}\left(\mathrm{SC}_{12} \mathrm{H}_{25}\right)_{y}$ and $\mathrm{Au}_{x}\left(\mathrm{SC}_{14} \mathrm{H}_{29}\right)_{y}$ systems have the same maximum emission value (corresponding to orange-red colour, $\lambda=633 \mathrm{~nm}$ ) when excited at $330 \mathrm{~nm}$ and $254 \mathrm{~nm}$, respectively. However, the quantum yield (QY) values of the two systems are quite different, and $\mathrm{Au}_{x}\left(\mathrm{SC}_{14} \mathrm{H}_{29}\right)_{y}$ shows higher QY value.

Figure 5 shows the PL spectrum of polystyrene-embedded Au clusters obtained from AuMMI. The strong fluorescence emission peak at wavelength $\lambda=520 \mathrm{~nm}$ leads to a yellow-green colour. The photoemission spectrum was recorded at an excitation wavelength $\lambda_{\text {exc }}=383 \mathrm{~nm}$.

The PL spectrum in Figure 6 shows the strong emission peak of alloyed $\mathrm{Au} / \mathrm{Ag}$ clusters. The excitation wavelength is $\lambda_{\text {exc }}=254 \mathrm{~nm}$ and the observed emission peak at wavelength $\lambda=710 \mathrm{~nm}$ corresponds to a large Stokes shift of $456 \mathrm{~nm}$. The emission peak of the PL spectrum of alloyed $\mathrm{Au} / \mathrm{Ag}$ clusters is clearly red-shifted. This red shift is found to increase with increasing the Ag content. Differently, the presence of MMI

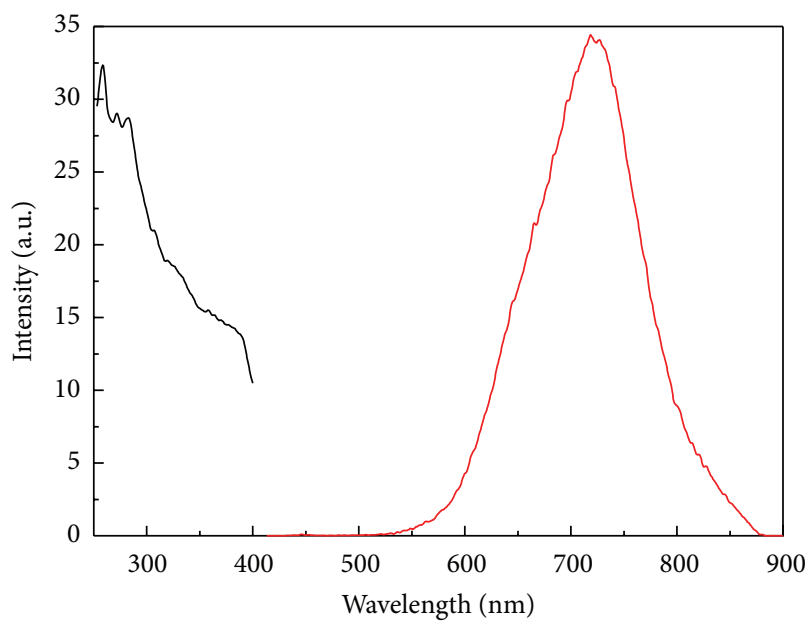

Figure 6: PL spectrum of polymer-embedded alloyed Au/Ag clusters.

on the surface of Au clusters allows for a blue shift of the emission peak. The large Stokes shift values observed for these special fluorescent systems can be ascribed to emissions involving gold electronic transitions from $s p$ to $d$ bands [14].

The QY value of thiol-derivatized gold clusters depends on the amount of charge transferred from the ligand to the metal core through the S-Au bonds during the excitation stage [14]. Therefore, it depends on the ability of the organic groups in the ligand molecule at the cluster surface to increase the electron density on the S atoms. Usually, the scarce electron-donating ability of alkyl groups (like, e.g., dodecyl- and terdodecyl-) results in a low QY of the resulting thiol-derivatized gold clusters. However, in the case of the $\mathrm{N}$-methyl-imidazole group, a completely different situation arises. In fact, in this case, the aromatic ring is rich in electron density, and it is able to donate electrons to the $S$ atom by the conjugation mechanism, thus increasing the charge 
transfer from the ligands to the metal core through the $\mathrm{S}-\mathrm{Au}$ bonds. This mechanism explains the observed high fluorescent quantum yield.

\section{Conclusions}

We have developed a simple synthesis method for producing different types of fluorescent $\mathrm{Au}$ and alloyed $\mathrm{Au} / \mathrm{Ag}$ clusters by mild thermal annealing of Au-thiolates and alloyed Au/Ag thiolate mixtures. The fluorescence emission was found to be tuned by (i) Au alloying with Ag (or other metals) and (ii) coating the Au cluster surface with aromatic molecules (i.e., 2-mercapto-1-methyl-imidazole) conjugated through the $\mathrm{S}$ atom. This novel class of high fluorescent species is extremely effective in lighting, lasers, and as luminescent probes in various technological applications (e.g., anticounterfeiting, downconversion, electroluminescence, analytical probes, biological labeling, etc.).

\section{References}

[1] W. Eberhardt, "Clusters as new materials," Surface Science, vol. 500, no. 1-3, pp. 242-270, 2002.

[2] J. D. Aiken III and R. G. Finke, "A review of modern transitionmetal nanoclusters: their synthesis, characterization, and applications in catalysis," Journal of Molecular Catalysis A, vol. 145, no. 1-2, pp. 1-44, 1999.

[3] D. I. Uhlenhaut, P. Smith, and W. Caseri, "Color switching in gold-polysiloxane elastomeric nanocomposites," Advanced Materials, vol. 18, no. 13, pp. 1653-1656, 2006.

[4] G. Carotenuto, A. Longo, P. Repetto, P. Perlo, and L. Ambrosio, "New polymer additives for photoelectric sensing," Sensors and Actuators B, vol. 125, no. 1, pp. 202-206, 2007.

[5] G. Carotenuto, G. L. Peruta, and L. Nicolais, "Thermo-chromic materials based on polymer-embedded silver clusters," Sensors and Actuators B, vol. 114, no. 2, pp. 1092-1095, 2006.

[6] G. Carotenuto, G. Pepe, D. Davino et al., "Transparentferromagnetic thermoplastic polymers for optical components," Microwave and Optical Technology Letters, vol. 48, no. 12, pp. 2505-2508, 2006.

[7] G. Carotenuto and L. Nicolais, Eds., Metal-Polymer Nanocomposites, John Willey \& Sons, Hoboken, NJ, USA, 2004.

[8] Z. Luo, X. Yuan, Y. Yu et al., "From aggregation-induced emission of $\mathrm{Au}(\mathrm{I})$-thiolate complexes to ultrabright $\mathrm{Au}(0) @ \mathrm{Au}(\mathrm{I})$ thiolate core-shell nanoclusters," Journal of American Chemical Society, vol. 134, pp. 16662-16670, 2012.

[9] G. Carotenuto, A. Longo, L. De Petrocellis et al., "Synthesis of molecular gold clusters with luminescence properties by mercaptide thermolysis in polymer matrices," International Journal of Nanoscience, vol. 6, no. 1, pp. 65-69, 2007.

[10] G. Carotenuto, M. L. Nadal, P. Repetto, P. Perlo, L. Ambrosio, and L. Nicolais, "New polymeric additives for allowing photoelectric sensing of plastics during manufacturing," Advanced Composites Letters, vol. 16, no. 3, pp. 89-94, 2007.

[11] J. Bomm, C. Günter, and J. Stumpe, "Synthesis and optical characterization of thermosensitive, luminescent gold nanodots," Journal of Physical Chemistry C, vol. 116, no. 1, pp. 81-85, 2012.

[12] G. Cardone, G. Carotenuto, P. Conte, and G. Alonzo, "Synthesis and characterization of a novel high luminescent gold-2mercapto-1-methyl-imidazole complex," Luminescence, vol. 26, no. 6, pp. 506-509, 2011.
[13] H. Schmidbaur, "The aurophilicity phenomenon: a decade of experimental findings, theoretical concepts and emerging applications," Gold Bulletin, vol. 33, no. 1, pp. 3-10, 2000.

[14] Z. Wu and R. Jin, "On the ligand's role in the fluorescence of gold nanoclusters," Nano Letters, vol. 10, no. 7, pp. 2568-2573, 2010.

[15] G. Carotenuto, L. Nicolais, and P. Perlo, "Synthesis of polymerembedded noble metal clusters by thermolysis of mercaptides dissolved in polymers," Polymer Engineering and Science, vol. 46, no. 8, pp. 1016-1021, 2006.

[16] G. Carotenuto, B. Martorana, P. Perlo, and L. Nicolais, "A universal method for the synthesis of metal and metal sulfide clusters embedded in polymer matrices," Journal of Materials Chemistry, vol. 13, no. 12, pp. 2927-2930, 2003. 

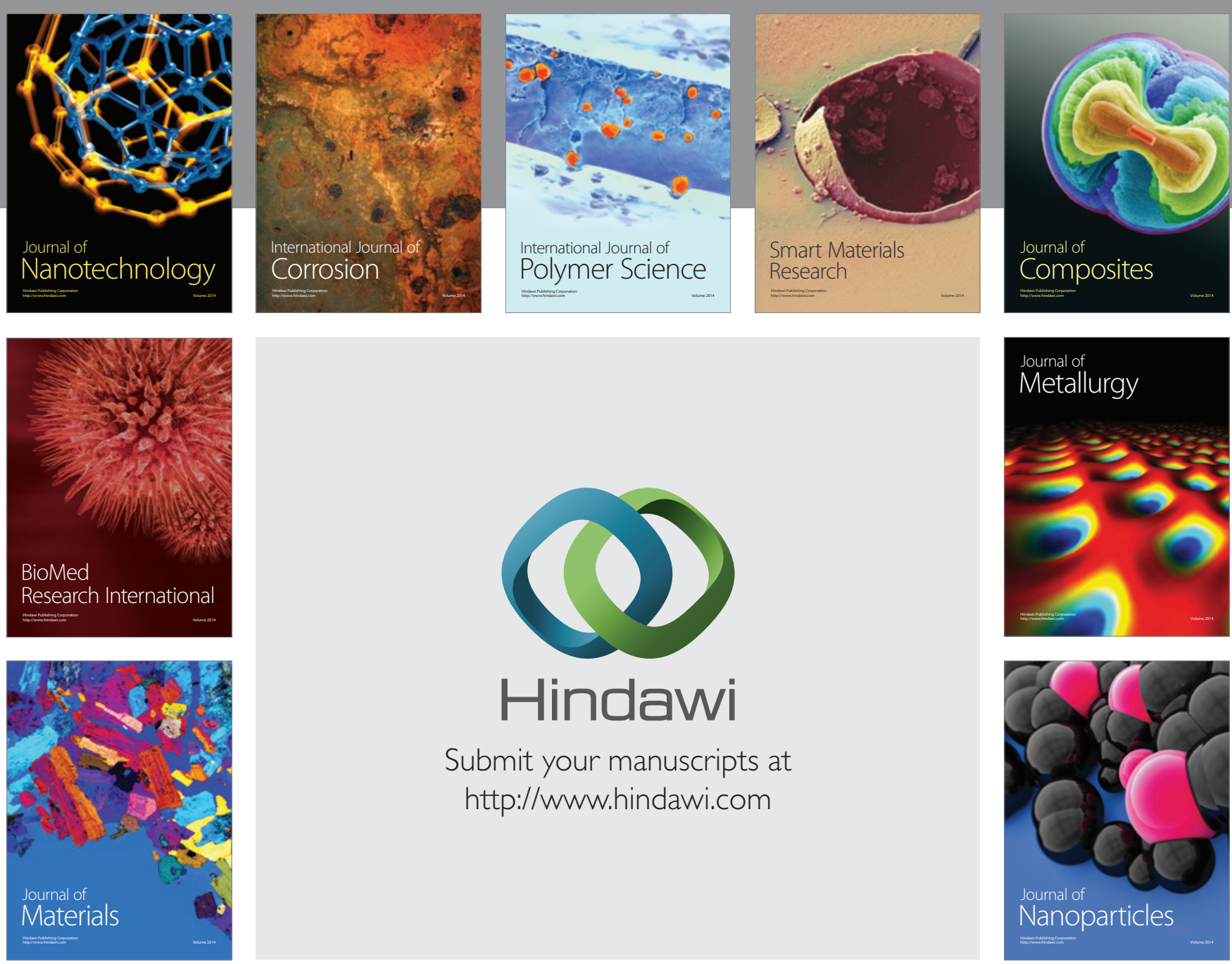

Submit your manuscripts at http://www.hindawi.com
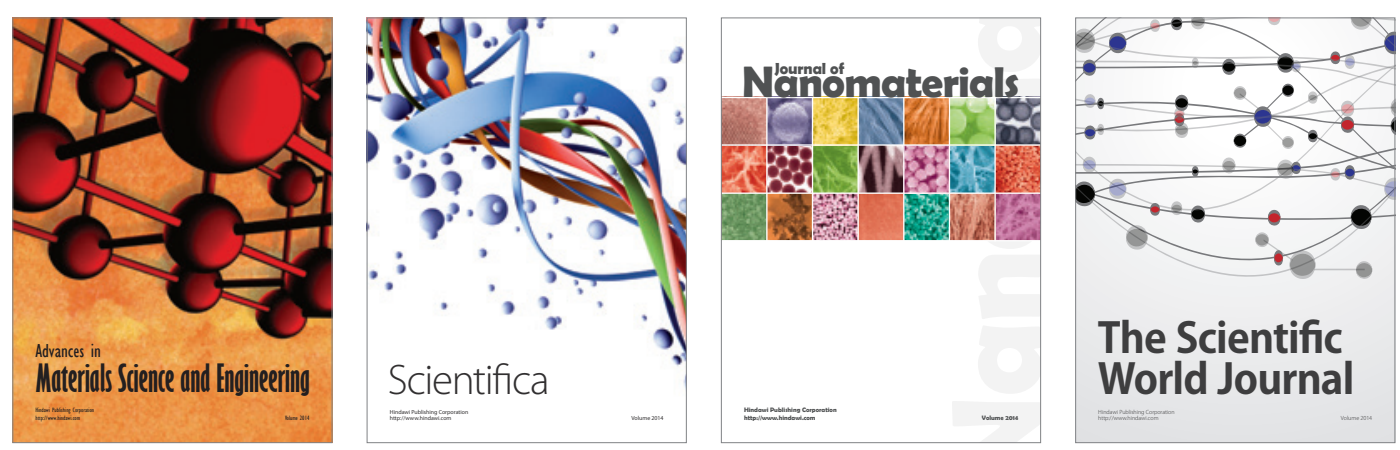

\section{The Scientific World Journal}
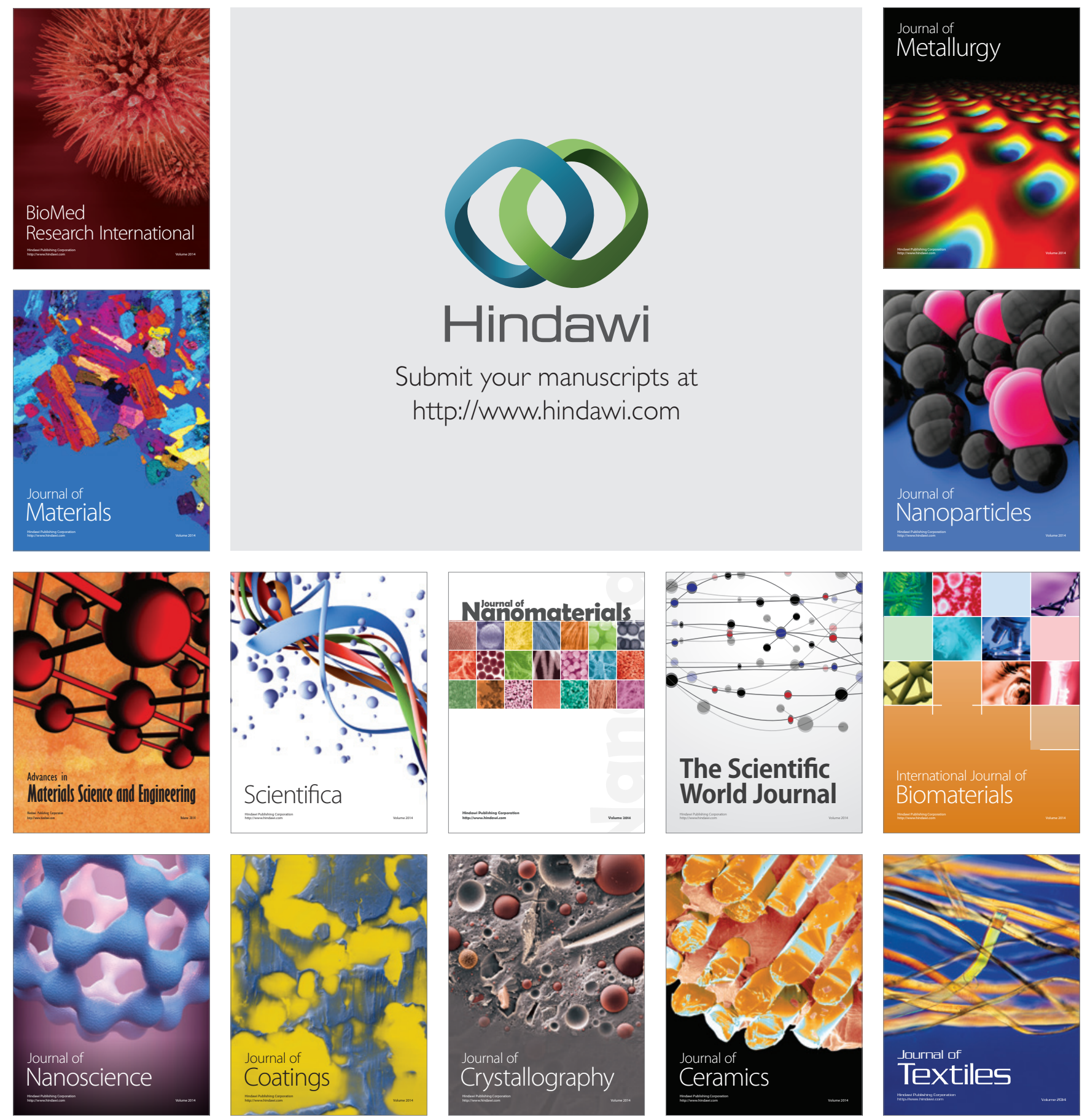